\title{
Finite-length effects on cylindrical Langmuir probes
}

\author{
Sigvald Marholm $\odot^{1, *}$ and Richard Marchand $\odot^{2, \dagger}$ \\ ${ }^{1}$ Department of Physics, University of Oslo, P.O. Box 1048 Blindern, N-0316 Oslo, Norway \\ ${ }^{2}$ Department of Physics, University of Alberta, Edmonton AB, Canada T6G 2E1
}

(Received 8 July 2019; accepted 8 January 2020; published 8 April 2020)

\begin{abstract}
Kinetic simulations are used to compute current characteristics of finite-length cylindrical probes, with particular attention to end effects. Currents collected per unit lengths, as a function of distance to the ends, are calculated and fitted to empirical analytic functions. These fits, in turn, can be interpolated and used to predict probe characteristics; that is, collected current as a function of applied voltage, for a broad range of physical parameters of relevance to laboratory and space plasma.
\end{abstract}

DOI: 10.1103/PhysRevResearch.2.023016

\section{INTRODUCTION}

Langmuir probes are amongst the most widely used instruments in laboratory and space plasma experiments. They work by exposing an electric conductor at a certain voltage to a plasma, from which they then collect current. The currentvoltage characteristics of the probe depends on many physical parameters, such as the electron density and temperature, the ion composition and temperature(s), the plasma flow velocity, the strength and direction of a magnetic flux density, exposure to solar radiation, and the proximity to other objects. Other parameters being known, and making use of a sufficiently accurate theoretical or computational model, it is possible to infer, for example, the plasma temperature and density, from probe characteristics [1-7].

Langmuir probes come in a variety of shapes, accompanied by analytical or empirical expressions for their characteristics, and they are used in different operational modes depending on what they are to measure. Of particular interest to us are thin cylindrical Langmuir probes (needle probes). A currentvoltage characteristic for idealized infinite probes can be derived analytically from orbital motionlimited (OML) theory, which suggests these probes are particularly well suited for electron density measurements $[1,8,9]$. Such probes are already employed in numerous space missions, of which can be mentioned the ICI [9] and ECOMA [10] sounding rockets, the NorSat-1 satellite [11], as well as CubeSats in the QB50 network of small satellites [12].

OML theory makes several assumptions, amongst them that the probe should be very thin, and very long compared to the Debye length. Finite-radius effects were studied numerically by Laframboise, and it is observed that the attracted-

\footnotetext{
*sigvald@marebakken.com; https://sigvaldm.github.io

${ }^{\dagger}$ rmarchan@ualberta.ca; https://sites.ualberta.ca/ rmarchan

Published by the American Physical Society under the terms of the Creative Commons Attribution 4.0 International license. Further distribution of this work must maintain attribution to the author(s) and the published article's title, journal citation, and DOI.
}

species current is virtually unaffected by these effects for cylinders of radius up to one Debye length (see Table 6c in [2]).

Finite-length effects, on the other hand, are a different matter. Cylindrical Langmuir probes are often equipped with a guard at one end, as illustrated in Fig. 1, which itself is attached to a supporting body such as a spacecraft bus $[3,5,12]$. The guard is ideally an extension of the cylindrical probe, having the same voltage as the probe, but being electrically insulated from it such that the current collected by the guard can be disregarded in the measurement. Edge effects due to the mounting point are then supposed to only affect the guard. Nevertheless, many practical probes still exhibit finitelength effects, which degrades the accuracy of the inferred plasma density, when based on the theory developed for an infinitely long cylindrical probe. Increasing the probe length will of course help, although there is an upper limit to what is practical. Besides, the literature reports different results on how long probes must be to overcome these effects, ranging from about 10 to more than 50 Debye lengths [8,13-16]. Some of these references also attempt to find expressions for the characteristics of the probes. However, present studies on finite-length effects are limited to very specific ranges of

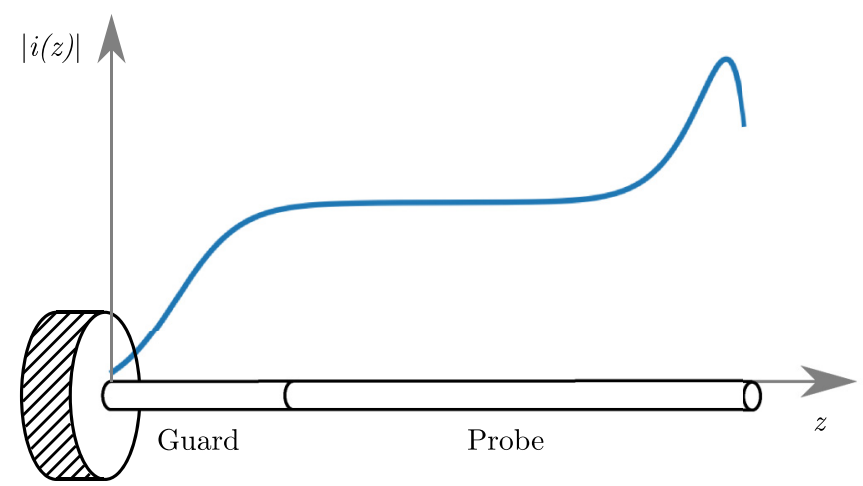

FIG. 1. Illustration of a Langmuir probe with guard mounted to a carrier object, with possible current collected per unit length $i(z)$ along the probe and guard. 
probe lengths, plasma parameters and/or experimental setup, which may explain the discrepancy between these studies; one laboratory experiment is performed using a conducting guard [13], the other with an insulating one [14]. Numerical experiments and analytical results often disregard the guard altogether, focusing on an idealized cylinder, possibly with some discussion on the expected changes due to the guard $[8,15,16]$. Clearly, there is a need for a more fundamental understanding of finite-length effects in cylindrical Langmuir probes.

In this paper, in order to better understand edge effects, we investigate the attracted-species current per unit length, $i(z)$, as a function of the position $z$ along a thin, cylindrical probe with both ends free. This approach is widely applicable: edge effects are clearly visible on $i(z)$, which can be used to evaluate how long a guard must be in order to mitigate edge effects. Moreover, by integrating $i(z)$ along the probe, a current-voltage characteristic can be obtained, and used to assess the applicability of the OML theory. If the probe has a guard, edge effects can be removed from one end of the function $i(z)$. A natural next step, which is not pursued here, would be to use the new characteristic to more accurately infer plasma parameters, for new as well as existing missions with available data. Through theoretical derivations and particlein-cell (PIC) simulations, we characterize the function $i(z)$ for thin, cylindrical probes of virtually arbitrary lengths, for normalized voltages up to 100 . The plasma is assumed to be collisionless, nonmagnetized, and nondrifting Maxwellian.

A possible application where the above conditions are well satisfied is to Langmuir probes on-board satellites and rockets at hundreds of kilometers altitude. In particular, we are interested in multineedle Langmuir probes (m-NLPs) [9]. The geometrical extent of these probes, including the sheath, is small enough compared to both the mean free path and the gyroradius of both electrons and ions that the plasma can be approximated as both collisionless and nonmagnetized, at least to first approximation [9,13]. Moreover, the m-NLP is operated exclusively at positive voltages with respect to the background plasma, such that electrons are always the attracted species and ions the repelled species. The plasma is typically streaming towards a satellite at roughly the orbital velocity of $v_{d} \approx 8 \mathrm{~km} / \mathrm{s}$. However, this drift velocity is orders of magnitude smaller than the thermal speed of the electrons, which have a temperature in the order of $1000 \mathrm{~K}$, and can therefore be neglected. While space plasmas are usually not in thermodynamic equilibrium, at mid latitudes, ionospheric electrons are generally well described by Maxwellian velocity distributions $[17,18]$.

As for the ions, they also have a temperature in the order of $1000 \mathrm{~K}$, which leads to a thermal speed that is much less than the drift velocity. However, it is easily demonstrated that the ions contribute negligibly towards the total collected current: a rough estimate of the ion current can be computed as the flux of ions streaming through the perpendicular cross section of the probe, $I_{i} \leqslant 2 r l q_{i} n_{i} v_{d}$, where $r$ and $l$ are the radius and length of the probe, respectively, and $q_{i}$ and $n_{i}$ are the charge and density of ions. The ions are singly charged oxygen of the same density as the electrons. The "less than" sign accounts for drift velocities not perpendicular to the probe as well as ion repulsion leading to a smaller effective cross section. For estimating the electron-to-ion current ratio $I_{i} / I_{e}$, the electron current $I_{e}$ may be approximated using OML theory, which should at least be accurate enough for the sake of this argument (in reality $I_{e}$ will be somewhat larger than predicted by OML theory). $I_{i} / I_{e}$ is then in the order of $1 \%$ for a probe voltage of $1 \mathrm{~V}$. The ion current can therefore be neglected, and the remaining electron current should be well described by the attracted-species current under the assumptions used in this paper. It is also worth noting that while other accurate instruments exists, the m-NLP offers exceptional spatial resolution while on board high-speed vehicles due to its high sampling frequency [9]. For satellites, the resolution is in the order of $10 \mathrm{~m}$. Efforts towards a better understanding of such probes are therefore most relevant.

The remainder of the paper is organized as follows. Following a review of some background equations in Sec. II, the theory is presented in Sec. III and the simulations characterizing $i(z)$, in Sec. IV. Several applications are briefly discussed in Sec. V, and finally the conclusion is given in Sec. VI.

\section{BACKGROUND}

It will be useful to briefly revisit OML theory [1]. Let us assume a collisionless, nonmagnetized, and nondrifting Maxwellian plasma with a species with charge $q$, mass $m$, density $n$, and temperature $T$. For a probe situated in this plasma with a voltage $V$ with respect to the background, we can define a normalized voltage

$$
\eta=-\frac{q V}{k T}
$$

where $k$ is Boltzmann's constant. According to OML theory, an infinite cylindrical probe would collect the following current from an attracted species, i.e., when $q V<0$ or $\eta>0$ [1]:

$$
\begin{aligned}
I_{\mathrm{OML}}(\eta) & =I_{\mathrm{th}}\left(\frac{2}{\sqrt{\pi}} \sqrt{\eta}+\exp (\eta) \operatorname{erfc}(\sqrt{\eta})\right) \\
& \approx I_{\mathrm{th}} \frac{2}{\sqrt{\pi}}(1+\eta)^{\frac{1}{2}},
\end{aligned}
$$

where the approximation on the second line is usually considered accurate for $\eta \geqslant 2$, and

$$
I_{\mathrm{th}}=\frac{q n v_{\mathrm{th}}}{\sqrt{2 \pi}} S
$$

is the current that would pass through the surface of the probe due to random thermal particle motion if it were at the same potential as the background plasma. $v_{\text {th }}=\sqrt{k T / m}$ is the thermal speed of the species, and $S=2 \pi r l$ is the surface area of the probe, with $r$ and $l$ being the probe radius and length, respectively. For repelled species, i.e., when $q V>0$ or $\eta<0$, the current collected is given by [1]

$$
I_{\text {ret }}(\eta)=I_{\text {th }} \exp (\eta) .
$$

It is remarkable that this latter equation is in fact true regardless of the shape of the probe (given the correct surface area $S$ ), including finite-length cylinders, and that the collected current is distributed evenly on the probe's surface [1]. We shall therefore limit our study on finite-length cylinders to the attracted-species current. For multiple species, the current 
TABLE I. Dimensions of relevant quantities. For example, the current per length $i$ is given in $\mathrm{A}^{1} \mathrm{~m}^{-1}$.

\begin{tabular}{lccccccccrr}
\hline \hline & $\mathrm{i}$ & $\mathrm{V}$ & $\mathrm{z}$ & $\mathrm{l}$ & $\mathrm{r}$ & $\mathrm{q}$ & $\mathrm{m}$ & $\mathrm{n}$ & $\mathrm{kT}$ & $\varepsilon_{0}$ \\
\hline $\mathrm{m}$ & -1 & 2 & 1 & 1 & 1 & & & -3 & 2 & -3 \\
$\mathrm{~kg}$ & & 1 & & & & & 1 & & 1 & -1 \\
$\mathrm{~s}$ & & 3 & & & & 1 & & & -2 & 4 \\
$\mathrm{~A}$ & 1 & 1 & & & & 1 & & & & 2 \\
\hline \hline
\end{tabular}

collected according to OML theory is the sum of the currents due to the different species.

\section{THEORY}

In the following, we are interested in determining the attracted-species current $i(z)$ per unit length along a cylindrical Langmuir probe of radius $r$ and voltage $V$ with respect to the background plasma. $z$ is the position along the probe, which stretches from $z=0$ to $z=l$. We assume an attracted species to be collisionless, nonmagnetized, nondrifting Maxwellian and uniform in the background. The species is then fully described by its charge $q$, mass $m$, background density $n$ and thermal energy $k T$, where $k$ is Boltzmann's constant and $T$ the temperature. Moreover, since this is an electrostatic problem, it is also reasonable to expect the vacuum permittivity $\varepsilon_{0}$ to enter the equations. We also assume, as in OML theory, that the current contributions due to different species can simply be superposed linearly. This means that the density and temperature of other species do not enter the equations of our attracted-species current (other species may well have different densities and temperatures). The attractedspecies current $i$ per unit length can then be described through a relation between all mentioned quantities:

$$
\mathcal{F}\left(i, V, z, l, r, q, m, n, k T, \varepsilon_{0}\right)=0 .
$$

The physical dimensions of the variables in this relation is given in Table I. Since this forms a $4 \times 10$ matrix of rank 4 , according to Buckingham's $\pi$ theorem [19], [20, pp. 22-26], the above relation can be written as a relation between $10-4=6$ dimensionless variables, which can be chosen freely as long as they are independent. We choose the normalized lengths

$$
\frac{z}{\lambda_{D}}, \quad \frac{l}{\lambda_{D}}, \quad \frac{r}{\lambda_{D}},
$$

where $\lambda_{D}=\sqrt{\varepsilon_{0} k T / q^{2} n}$ is the attracted-species Debye length, the normalized voltage and current,

$$
\frac{q V}{k T}, \quad \frac{i}{i_{\text {th }}},
$$

where $i_{\text {th }}=I_{\mathrm{th}} / l=q n r \sqrt{2 \pi k T / m}$, and finally, the plasma parameter,

$$
n \lambda_{D}^{3}
$$

Equation (6) can thus be reduced to

$$
\mathcal{G}\left(\frac{i}{i_{\mathrm{th}}}, \frac{q V}{k T}, \frac{z}{\lambda_{D}}, \frac{l}{\lambda_{D}}, \frac{r}{\lambda_{D}}, n \lambda_{D}^{3}\right)=0 .
$$

We shall limit the discussion to thin probes, $r / \lambda_{D} \rightarrow 0$, and weakly coupled plasmas, $n \lambda_{D}^{3} \rightarrow \infty$, and can therefore disregard the latter two variables. According to Laframboise [2] the first assumption is well justified for infinitely long probes when $r / \lambda_{D}<1$. It is reasonable to assume that the same holds for finite-length probes. Equation (7) can then be inverted with respect to the first argument to yield

$$
i(z)=i_{\mathrm{th}} G\left(\frac{z}{\lambda_{D}} ; \frac{l}{\lambda_{D}},-\frac{q V}{k T}\right),
$$

where $G$ is a hitherto unknown function. Alternatively, one can extract a factor from $G$, allowing the expression to be rewritten as a modification to the OML theory,

$$
i(z)=i_{\mathrm{OML}} g\left(\frac{z}{\lambda_{D}} ; \frac{l}{\lambda_{D}},-\frac{q V}{k T}\right),
$$

where $i_{\mathrm{OML}}=I_{\mathrm{OML}} / l$, and $I_{\mathrm{OML}}$ is given by Eq. (2). We refer to $G$ and $g$ as normalized current profile functions or just profile functions, and while either can be used, we use $g$ for easier comparison with OML theory. Note that the use of $g$ do not rely on the correctness of OML theory, since the OML current merely appear as a normalization. For convenience, we introduce the following dimensionless variables:

$$
\zeta=\frac{z}{\lambda_{D}}, \quad \lambda=\frac{l}{\lambda_{D}}, \quad \eta=-\frac{q V}{k T} .
$$

Given Eq. (9), the problem has been reduced from finding a relation between all quantities in Eq. (6) to characterizing the function $g(\zeta ; \lambda, \eta)$.

Before characterizing the profile function, we shall make a few theoretical predictions on its shape. First, due to the geometrical symmetry of the problem, it must be left-right symmetric about the center of the probe, $g(\lambda-\zeta)=g(\zeta)$. Second, suppose the probe is lengthened until a section emerges in the middle, from which the edges cannot be "seen." In this region $g$ will necessarily be flat, and equal to the value of an infinitely long probe. We can define this value rigorously as

$$
C(\eta)=\lim _{\lambda \rightarrow \infty} g(\lambda / 2 ; \lambda, \eta) .
$$

For our model to comply with OML theory, $C$ should equal 1. However, we do not enforce compliance with OML theory, but instead treat $C$ as a coefficient to be determined. This allows the degree of compliance to be measured as the deviation of $C$ from 1. Third, when the flat region of $g$ emerges, the edge effects no longer overlap. That is, any point on $g$ close enough to either end to experience edge effects do not "see" the other end, and as such is independent of the distance to it. As a consequence, the shape of the edge effects near either end remains the same as the probe is further extended to arbitrary lengths.

\section{SIMULATIONS}

The characterization of $g(\zeta ; \lambda, \eta)$ is constructed from simulation results obtained with PTETRA [21,22], an electrostatic PIC code in which space is discretized with unstructured tetrahedral cells. PTETRA records the current density through the probe surface by counting the number of particles passing 


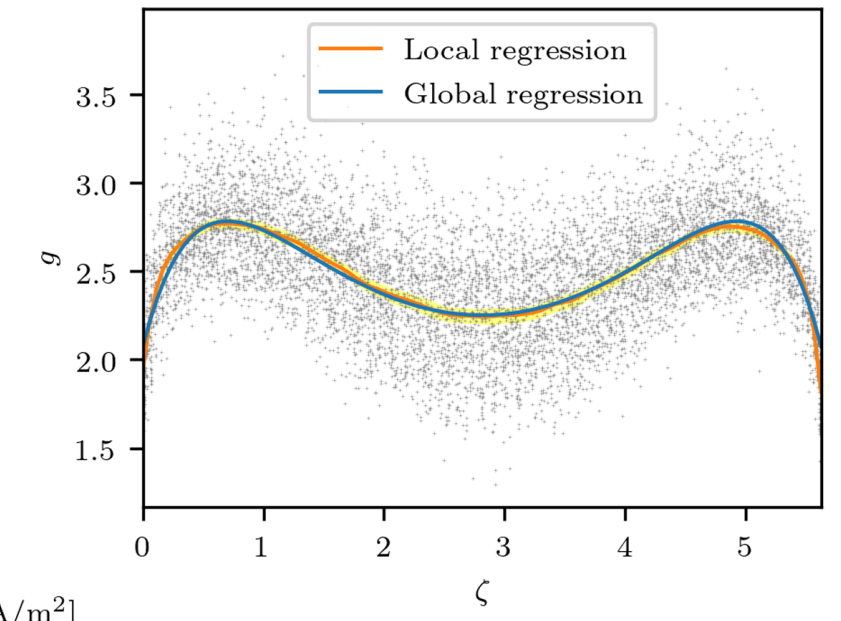

$\left[\mathrm{A} / \mathrm{m}^{2}\right]$

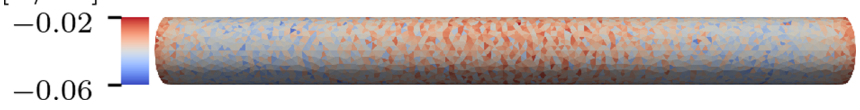

FIG. 2. (Bottom) Surface current density for a probe with $\lambda=$ 5.63 and $\eta=25$. The current through each facet is averaged with a relaxation time of $1 \mu \mathrm{s}$. (Top) Data points $\left(\zeta_{i}, g_{i}\right)$ obtained from the facet currents (gray dots), as well as a nonparametric local regression (orange) and a parametric global regression through these data points (blue). The barely visible yellow band shows a pointwise 99\% confidence interval on the local regression.

each boundary facet every time step. An example of this simulated surface current density can be seen in the lower part of Fig. 2, which is for a probe with $\lambda=5.63$ and $\eta=25$. This can then be used to infer $g(\zeta ; \lambda, \eta)$, as represented by a curve fit, for these particular values of $\lambda$ and $\eta$. In order to fully characterize $g$, we have to do a sweep of simulations for different values of $\lambda$ and $\eta$. Interpolation of the fitting coefficients is used to obtain $g$ between the simulated values of $\lambda$ and $\eta$.

\section{A. Simulation setup}

We simulate a plasma consisting of two species, electrons and singly charged ions. Both species are nonmagnetized, nondrifting Maxwellian and have a density of $3.5 \times 10^{11} \mathrm{~m}^{-3}$ and a temperature of $0.08 \mathrm{eV}$ for all simulations, yielding an electron Debye length of approximately $3.55 \mathrm{~mm}$. Recall that this arbitrary choice is by no means limiting, since $g$ does not depend upon density and temperature independently, but only on its arguments $\zeta, \lambda$ and $\eta$.

Simulations have been run for probes of length $(l) 2,5,10$, $20,30,40,80,200,400,1000,2000 \mathrm{~mm}$ and in each case, for voltages $(V) 0.16,0.48,0.8,1.36,2,2.56,4,6,8 \mathrm{~V}$, in total 99 simulations. The lengths and voltages were chosen heuristically to cover realistic probes, span a wide parameter range, and have a finer representation in regions where the fitting coefficients of $g$ change more rapidly. Notice also that for these voltages, the electron current is the attracted-species current.

Since Laframboise [2] found finite-radius effects to be negligible for radii up to the Debye length for infinite-length cylinders, we have chosen a probe radius of $1 \mathrm{~mm}$, well below the Debye length. The cylindrical probe is centered inside a cylindrical simulation domain of radius $40 \mathrm{~mm}$ and a length extending $40 \mathrm{~mm}$ beyond the probe in the $\pm z$ directions. It is important that the outer boundary be sufficiently far away from the probe, since the potential is set to $0 \mathrm{~V}$ there (Dirichlet boundary conditions). The mesh is generated using Gmsh [23], with a resolution of $6 \mathrm{~mm}$ on the outer boundary and $0.2 \mathrm{~mm}$ on the probe surface. Extensive experiments were carried out with different domain sizes and resolutions prior to settling at these values, to ensure the results are as accurate as practically possible.

PTETRA automatically computes a suitable time step that resolves the plasma period, as well as being small enough that a typical particle trajectory does not cross more than one Voronoi cell in any given time step. The time step also accounts for possible increases in particle energies and speeds near objects biased to various potentials.

Whereas the profile function $g$-representing the attractedspecies current - should be independent of the mass of the repelled species (ions), PTETRA does not discriminate species when recording surface currents. It is therefore important that we reduce the ion current in the simulations to an acceptably low level by having sufficiently massive ions. We have chosen to use an ion mass of 1 atomic mass units (hydrogen ions) for $\eta<10$, and a reduced mass of $1 / 16$ atomic mass units for $\eta \geqslant 10$, where the ions are more strongly repelled. Using artificially low mass ions in the simulations has the advantage of reducing the time needed to reach steady state, while having a negligible effect on the collected currents. Indeed, using OML theory as an order-of-magnitude estimate (see Sec. II), the resulting repelled-species current should be less than roughly $0.2 \%$ of the attracted-species currents. Whereas we run the $\eta<10$ simulations to $40 \mu$ s to reach a steady state, the higher voltage simulations only need to run $10 \mu \mathrm{s}$ due to the lower ion mass, which is convenient, since these simulations have a smaller time step to account for more energetic electrons near the probe.

As is a standard practice in PIC simulations, we employ simulation particles that correspond to multiple physical particles in order to reduce the cost of the simulations. In each simulation, we prefill the domain uniformly with 50 million simulation particles of each species, meaning that each simulation particle corresponds to between 2.9 and 73 physical particles depending on the size of the domain (which depends on the probe length). Experiments were also carried out with different amounts of simulation particles to verify that these values were indeed sufficient. It is also worth noting that the current density through the probe is averaged with a relaxation time of $1 \mu \mathrm{s}$ according to the relaxation scheme in [21].

\section{B. Selected results}

An example from one of our simulations is shown in Fig. 2. The lower part shows the probe surface, with the current density through each facet. The current density at each facet is then multiplied by $2 \pi r$ to get current per unit length, and then divided by $i_{\mathrm{OML}}$ to get data points for the profile function as indicated in the scatter plot. Currents from the circular faces at the ends of the probe are excluded. Clearly, there is a significant amount of particle noise which is not part of the underlying profile function. From the dimensional argument 

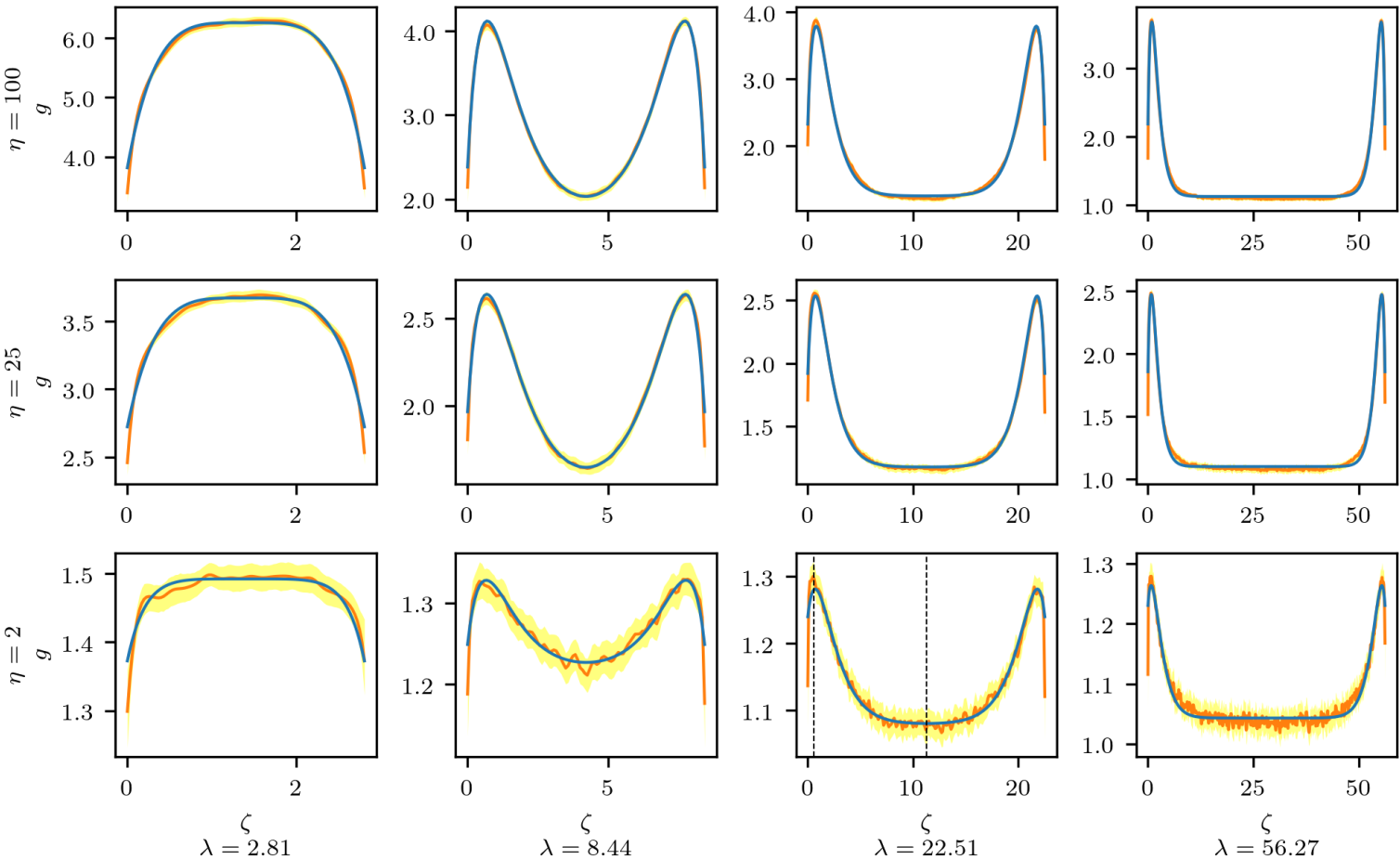

FIG. 3. Local (orange) and global (blue) regressions for selected simulations. The yellow band is the pointwise $99 \%$ confidence interval of the local regression. The vertical dashed lines indicate the position of the test-particle simulations.

in Sec. III, the profile function cannot vary on spatial scales much smaller than the Debye length. To smooth out this noise without making any assumption on the form of the profile function (to perform a nonparametric regression), we use a local quadratic regression with a Gaussian weighting window [24, pp. 191-199]. The window has a standard deviation of 0.1 Debye lengths in order not to suppress actual variations in the profile function. Local polynomial regression is superior compared to for instance a moving average in that it does not underestimate the slope near the edges. Note that we do not employ the popular method for enhanced robustness described in Ref. [25], since in our case this mostly treats higher-valued datapoints as outliers, leading to a small negative bias in the obtained profile function. The global regression also seen in Fig. 2 will be explained in Sec. IV C

The barely visible yellow band in Fig. 2 is a pointwise 99\% confidence interval of the regression, created by performing local regression on 2000 bootstrap datasets [24, pp. 249-250] and taking the middle $99 \%$ as the confidence interval. Bear in mind that this is only an estimate of the distribution of data points mapped through the local regression operation. It neither accounts for errors due to an incorrectly applied window nor errors in the underlying PIC simulation.

Figure 3 shows the profile function for a selection of probe lengths and voltages. The profile functions feature a characteristic peak near each end of the probe, and the peaks' magnitudes increases with increasing voltage (mind the axes). For shorter probes, the peaks merge into one another, whereas for longer probes, there is a flat mid-section which approaches the value predicted by OML theory for an infinitely long probe. The simulations with $\eta=2$ exhibit more noise than the other simulations, even after the local regression. This is reasonable, given that a probe with a lower potential attracts fewer electrons, i.e., collects less current, and that the signalto-shot noise ratio is proportional to the square root of the current [26], [27, pp. 475-476].

VTK files with surface current densities are made publicly available for all 99 probe simulations [28], as is the computer program LOCALREG [29] used to perform local regressions.

The characteristic profile of $g(\zeta)$ can be understood from the velocity distribution of particles at selected points along the cylinder. This is illustrated in Fig. 4 where cross sections of the particle velocity distribution function $f$ at the probe surface $(x=0, y=1 \mathrm{~mm})$, are plotted at $\zeta=0.56$ and 11.25; the former corresponding to the left peak in Fig. $3(\eta=$ $2, \lambda=22.51)$, and the latter, to the middle of the probe. These positions are identified with two vertical lines in the third panel from the left, in the third row in Fig. 3. The cross sections, corresponding to the $v_{x}=0$ plane, was chosen so as to illustrate the left-right asymmetry near the left end of the probe. Distributions were calculated using Liouville's theorem for the one-particle distribution function in a collisionless plasma, and particle backtracking [30]. The figure shows the distribution function multiplied by the thermal speed to the third power, $f \times v_{\mathrm{th}}^{3}$, while the velocity coordinates $v_{y}$ and $v_{z}$ are normalized to the thermal speed.

The peak in the collected current density at $\zeta=0.56$ is consistent with the larger extent of the distribution function for $v_{z}>0$, which in turn corresponds to particles coming from the left and, for an infinite probe, would have been collected farther to the left. Indeed, in that case, particles approaching from the left with a velocity nearly parallel to the probe axis would be collected to the left of $\zeta=0$. Moreover, particles grazing the end of the probe from below can be deflected by 

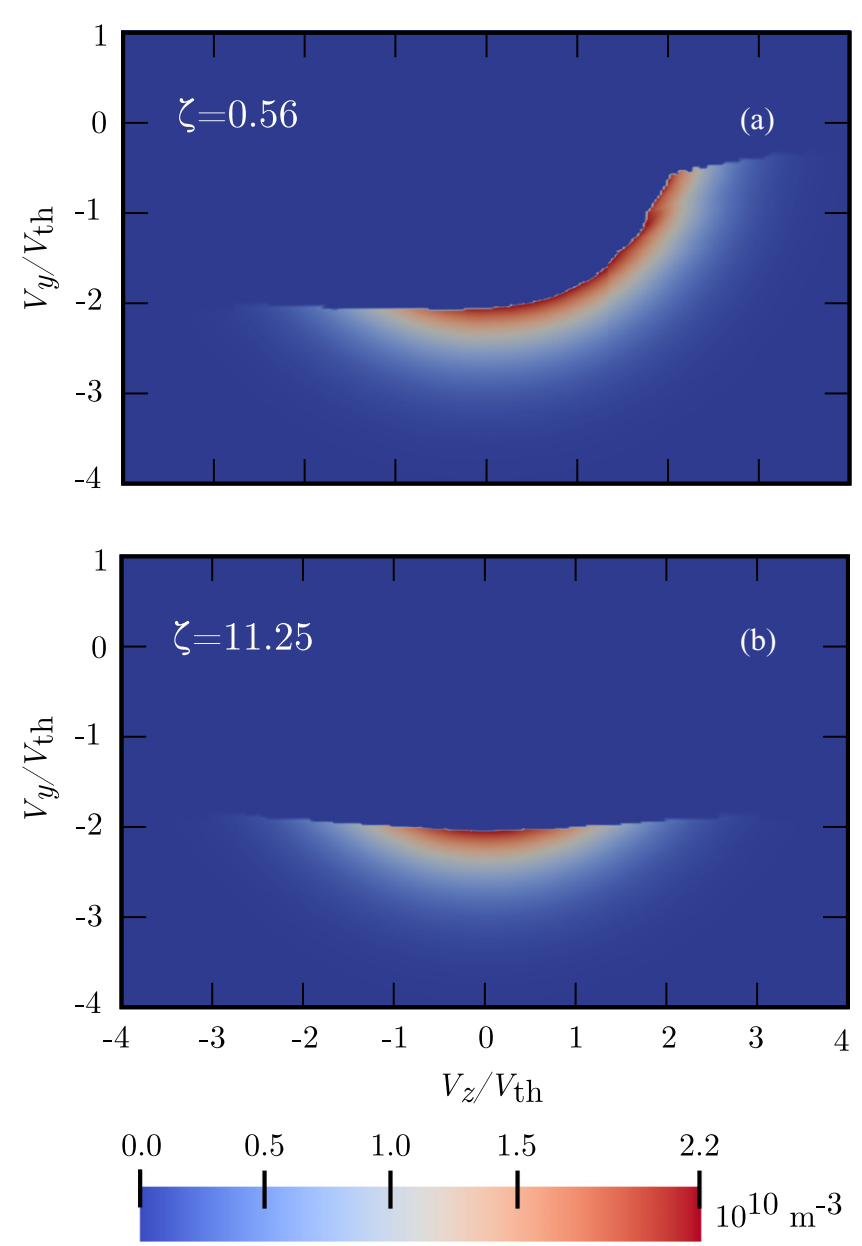

FIG. 4. Velocity distribution for particles at the surface of the probe $(x=0, y=1 \mathrm{~mm})$ for two positions along the $z$ axis. The top panel $(\zeta=0.56)$ shows the distribution at the left maximum in $g$, while the bottom panel $(\zeta=11.25)$ shows the distribution in the middle of the probe.

the attractive sheath electric field, and be collected at $y=1$ $\mathrm{mm}$ near the edge, while with an infinite probe, they would have been collected below the probe and to the left of $\zeta=0$. The energization of particles collected at the probe is clearly seen in Fig. 4. The background nondrifting Maxwellian electron distribution function assumed in the simulations has its maximum at $\mathbf{v}=\mathbf{0}$, and it is spherically symmetric in velocity space. At the probe, all particles must have an energy of at least $\eta k T=0.16 \mathrm{eV}$, corresponding to a normalized speed of 2. In particular, the maximum of the distribution function, corresponding to particles at rest away from the probe, is exactly at normalized speed of 2 in both figures, and it marks the boundary between where $f$ vanishes identically $\left(v / v_{\text {th }}<\right.$ $2)$, and where it has nonzero values $\left(v / v_{\text {th }} \geqslant 2\right)$. In the upper panel of Fig. 4, the extension to the right in the circle arc boundary is due to particles coming from the left, that would have been collected elsewhere in an infinitely long cylinder, as explained above. In the lower panel, the boundary in $f$ is nearly straight at $v_{y} / v_{\text {th }}=-2$, but a close look reveals a small upward curvature due to finite-length effects. In this case, with the value of $\lambda$ and $\eta$ considered, it is possible for particles with the right energy and grazing incidence coming from below on either side, to be deflected by the sheath, and be collected at the probe center. It should also be noted that the boundary between $f=0$ and $f>0$ in the figure is not perfectly smooth as one might expect analytically. This is due in part to the discretization of $f$ on a finite grid, and the discretization of the cylinder in terms of triangular facets.

\section{Curve fits}

As mentioned in Sec. IV A, many more simulations (99 in total) were made than illustrated in Sec. IV B. In all cases, similar basic profiles were found as illustrated in Fig. 3. With shorter probes, with small values of $\lambda$, there is strong overlap between end effects, $g(\zeta)$ has a single maximum at the probe center, and a monotonic decrease toward the ends. As $\lambda$ increases, the characteristic central hump progressively splits, leading to two humps that will remain near each end. It turns out that the profile function, as indicated by the nonparametric local regression in Fig. 3, can be parametrized rather well with the following analytic expression:

$$
\tilde{g}(\zeta)=C(1+\tilde{h}(\zeta)+\tilde{h}(\lambda-\zeta)),
$$

where

$$
\tilde{h}(\zeta)=A\left(\zeta-\delta+\alpha^{-1}\right) \exp (-\alpha \zeta)
$$

$\tilde{g}(\zeta)$ is constructed so as to be left-right symmetric about the probe center. In addition, each $\tilde{h}$ function describes the edge effects due to one end, approaching zero far away from its respective end. For long probes, $\delta$ is the distance from either end to the peak in $\tilde{g}$ (this interpretation is the reason for including the $\alpha^{-1}$ term). Further on, $\alpha$ relates to how fast the edge effects decay when moving inwards from the peak and $A$ relates to the amplitude of the peaks, the amplitude being $A \alpha^{-1} \exp (\alpha \delta)$. Due to the normalizations, all coefficients are expected to be of order unity. The fitting coefficients $C, A, \alpha$, and $\delta$ are determined using a weighted nonlinear least squares method, i.e., by minimizing the sum of weighted squared residuals,

$$
\sum_{i=1}^{N} w_{i}\left[g_{i}-\tilde{g}\left(\zeta_{i}\right)\right]^{2},
$$

where $\left(\zeta_{i}, g_{i}\right)$ are the data points in the scatter plot, $w_{i}$ the weight assigned to each data point and $N$ the number of data points/facets. As the probe lengthens, a decreasing fraction of the data points will be within the peak region near the edges, and if equal weights were used, the algorithm would fail to capture the peaks near the edges for long probes with small voltages. To correct this, at least half of the total weight is spent on the $N_{e}$ data points within 5 Debye lengths of either edge. More precisely, if $w_{e}$ is the weight of the $N_{e}$ data points within 5 Debye lengths of either edge, and $w_{m}$ is the weight of the $N_{m}$ remaining data points in the mid-section, the weights satisfy the following equations:

$$
\begin{aligned}
\sum_{i=1}^{N} w_{i} & =N_{m} w_{m}+N_{e} w_{e}=1, \\
N_{e} w_{e} & =\max \left(\frac{N_{e}}{N}, 0.5\right) .
\end{aligned}
$$




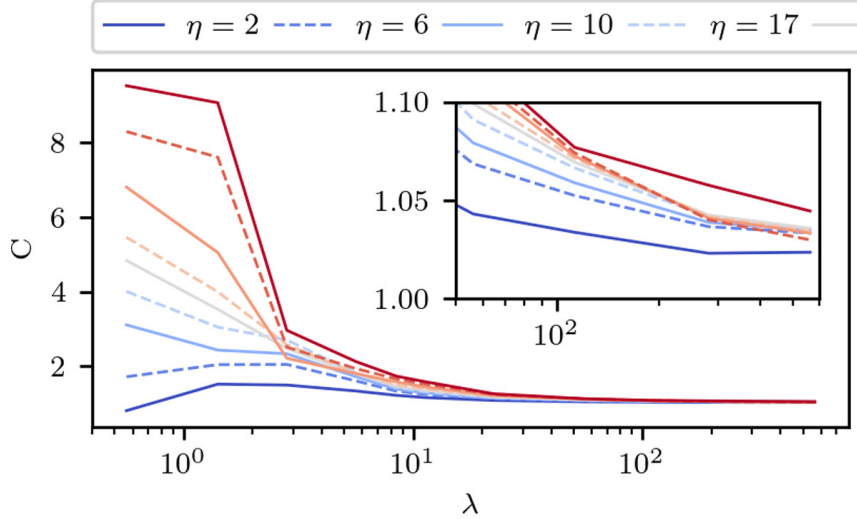

(a) C-coefficient

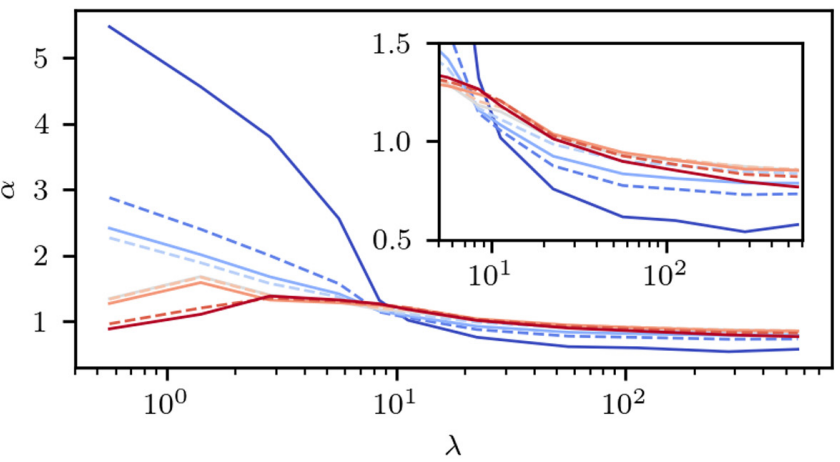

(c) $\alpha$-coefficient

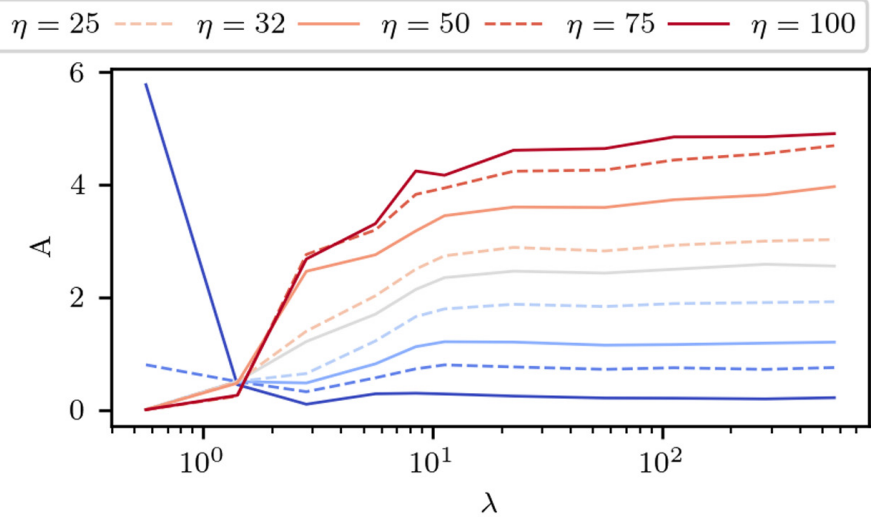

(b) A-coefficient

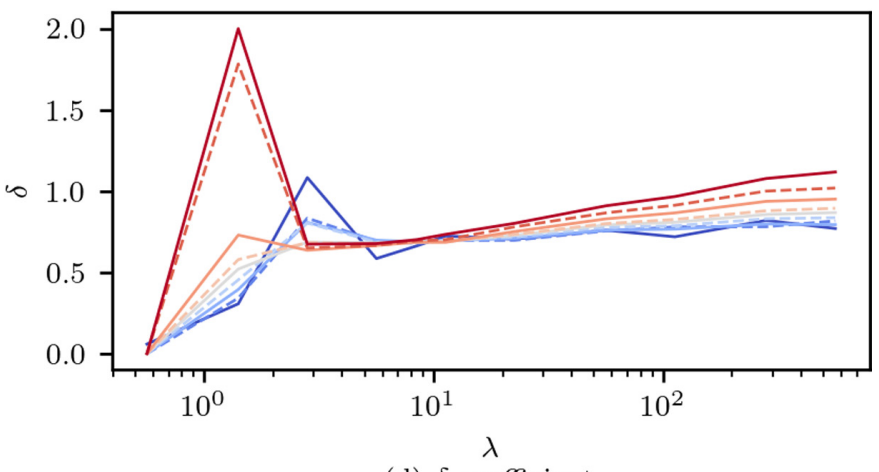

(d) $\delta$-coefficient

FIG. 5. Fitting coefficients of the expression Eq. (12) to the 99 probe simulations. Coefficients for $\lambda<3$ are considered less reliable.

The max operator prevents $w_{e}<w_{m}$ for $\lambda<20$ by making the scheme reduce to a nonweighted least squares method.

The fitting coefficients are illustrated graphically in Fig. 5. For $\lambda>3$, the coefficients form more or less smooth surfaces, which can then be used to do interpolation. For the three shortest lengths, i.e., $\lambda<3$, the coefficients are less wellbehaved. In addition, it was necessary to manually bound some of the coefficients for these simulations, since there are several minima in the sum of squared residuals. Coefficients for $\lambda<3$ should therefore be regarded as less reliable, and used with caution.

Since the coefficients are determined independently for each simulation, they are in effect functions of $\lambda$ and $\eta$ as independent variables. It is interesting to observe that as $\lambda$ increases, however, all the fitting coefficients asymptotically approaches a value dependent only on $\eta$. This is not a coincidence; since each $\tilde{h}$ function describes the edge effects due to one end, its shape will necessarily become independent of $\lambda$ for sufficiently long probes, when edge effects do not overlap, as described in Sec. III. This happens at $\lambda \sim 300$. It follows that the coefficients can be extrapolated to arbitrary lengths, by using the right-most values as the asymptotic values. The model can thus be applied to probes of arbitrary length, all the way down to below the Debye length.

As for the probe potential, we have simulated the range $\eta \in[2,100]$. It further follows from the definition of $g$ and the thermal current $i_{\text {th }}$ that $g(\zeta ; \lambda, 0)=1$ everywhere. This can be represented in terms of the parametrization Eq. (12) by setting $C=1$ and $A=0 . \alpha$ and $\delta$ are not uniquely defined, but we choose them as for $\eta=2$ to provide for smooth interpolation, and add data-points such as to make the grid of coefficients rectangular and structured. With this, the model covers the range $\eta \in[0,100]$. For $\eta<0$, Eq. (5) can be used.

Numerical values of the coefficients are included in the dataset along with the VTK files [28]. Moreover, current profiles and integrated total currents can be programmatically accessed through the Langmuir library [31], in which we have implemented linear interpolation between the coefficients, as used for the remainder of this paper. We have also made use of $[32,33]$.

While it is visually evident from Fig. 3 that the parametrization is quite good, it is of interest to quantify the errors of the fits. However, due to the large particle noise, the sum of squared residuals [Eq. (14)] is a large number and is not representative of the statistical errors in the fits. The coefficient of determination (often referred to as $R^{2}$ ) is also not suitable, due to the nonlinearity of the fit. Instead, we report two measures. The first is the error in the total current collected by the probe. The total current collected by the probe can be computed from the fitted expression $\tilde{g}$ as

$$
I=\int_{0}^{l} i(z) d z=i_{\mathrm{OML}} \lambda_{D} \int_{0}^{\lambda} \tilde{g}(\zeta) d \zeta .
$$

This current agrees with the total current collected by all facets of the probe (excluding the circular end faces) within $0.4 \%$ for all 99 simulations. The smallness of the error according to this measure is certainly desirable. Nonetheless, it does not say much about how well the shape of $\tilde{g}$ matches the true $g$. The second measure is therefore a relative $L_{2}$ error 
norm against the local regression $\bar{g}$, defined as follows:

$$
\sqrt{\frac{\int_{0}^{\lambda}(\tilde{g}-\bar{g})^{2} d \zeta}{\int_{0}^{\lambda} \bar{g}^{2} d \zeta}} .
$$

The integrals were evaluated numerically using the mid-point rule $[34$, p. 286] with a step-size $\Delta \zeta=0.02$. For the shortest probes $(2 \mathrm{~mm})$, this error is up to 0.05 and the fit visibly deviates from the local regression. For all other probes, the error is below 0.03, and there are small visible deviations. It should be understood that this error is not all in $\tilde{g}$, but also includes leftover noise in the local regression $\bar{g}$ that is more effectively filtered out by $\tilde{g}$. The fact that the fits are so good, while there is still some irregularities in the coefficients in Fig. 5, indicate that the fits are not very sensitive to the exact values of the coefficients.

It is also of interest to see how the model compares to OML theory. Since $g \rightarrow C$ as $\lambda \rightarrow \infty$ and $C$ is between 1.00 and 1.05 for all $\eta$ for the largest value of $\lambda$, our model is within $5 \%$ of OML theory. The error is in part due to inaccuracy in the PIC simulations, and in part, to the fitted expression $\tilde{g}$ 's inability to exactly match the flat, middle part of $g$, as witnessed in Fig. 3. These errors are believed to be roughly equal contributors, and as such the error of the PIC simulations is believed to be accurate within a few percent. Considering $\delta$, when $\lambda$ is sufficiently large and the peaks do not merge together, the peaks are always roughly 1 Debye length from the edges; a little more for the higher voltages, and a little less for lower ones.

\section{APPLICATIONS AND DISCUSSION}

In this section, we briefly describe possible applications of the results presented above.

\section{A. Finite-length probe characteristics}

It can be seen from Eq. (2) that the current collected by an infinitely long cylindrical probe can be written as a power law:

$$
I \propto I_{\mathrm{th}} \eta^{\beta},
$$

with $\beta=0.5$ for $\eta \gg 1$. It turns out that this expression also holds true for spherical probes but with $\beta=1$ [1]. For this reason, it has several times been assumed that this expression will also hold for thin, finite-length cylindrical probes, approaching 0.5 as the length increases and 1 as it decreases and the probe approaches the shape of a small grain $[13,15,16]$.

With our model, we can compute the characteristics for a probe by evaluating Eq. (16) for a sweep of voltages $\eta \in$ $[10,100]$. Figure 6 shows the characteristics obtained for five different probe lengths. We remark that the interpolation is not linear in $I$ but in the coefficients, and that this may lead to irregular behavior for short probes where the coefficients changes more rapidly. This is seen for the case $\lambda=1$. An alternative approach could be to compute $I$ on the grid of $(\lambda, \eta)$ values for which simulations were carried out, and then interpolate $I$.

We also made a least squares curve fit to Eq. (18) in order to test the power-law hypothesis, as indicated by dotted lines. The estimated $\beta$ values are also shown in the figure. It is clear

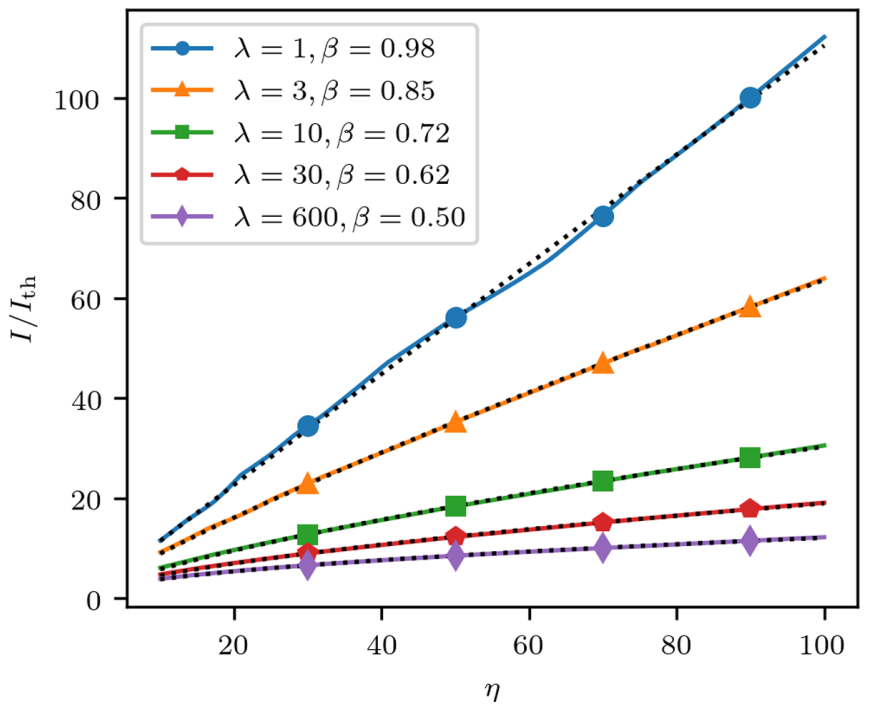

FIG. 6. Characteristics for probes of varying lengths, and estimated $\beta$ coefficient. Note that the characteristics are in effect normalized by length since $I_{\text {th }}$ includes the length [see Eq. (4)].

that the power law is indeed a very good approximation, and moreover, that $\beta$ behaves as expected. One should remember, however, that for a practical probe $\beta$ is not constant. As the density and temperature vary, the probe length-to-Debye length $\lambda$ changes, and $\beta$ is a function of $\lambda$. Recall also that the current collected by the circular end faces is not included in the integrated expression, and that this may change the characteristics for the shortest probes somewhat.

\section{B. Guarded probes}

Until now, we have mainly been concerned with probes with both ends free. The current collected near the end will certainly be altered in a nontrivial way when the probe is attached to some other, arbitrary object. To get a more predictable behavior, it is customary to use a "guard" which should ideally be an extension of the cylindrical probe, and which often has the same potential as the probe but is not in direct contact with it, such that the current collected by the guard can be excluded from measurements [3,5,12]. This is believed to eliminate end effects near the guard. In practice, the guard usually has a slightly larger radius, and there is an insulating transition in-between the guard and the probe, but these differences are kept as small as possible such that they can be ignored, at least to first approximation.

There are two ways of accounting for a guard with our model. The first approach is to let $\lambda=\lambda_{g}+\lambda_{p}$ where $\lambda_{g}$ is the length of the guard, and $\lambda_{p}$ is the length of the actual probe. The current collected by the probe can then be computed similarly as in Eq. (16), except that the integration should start at a lower limit $\lambda_{g}$. Figure 7 shows the current profile for a probe with $\lambda_{p}=30$ and $\eta=10$ both without a guard $\left(\lambda_{g}=0\right)$ and with a finite-length guard with $\lambda_{g}=5$. The dotted lines indicate the part excluded from the integration. Notice also that the profile function $\tilde{g}$ (or even just the $\tilde{h}$ part of it) can be used as a first approximation on how long the guard must be in order to suppress edge effects to a certain level. 


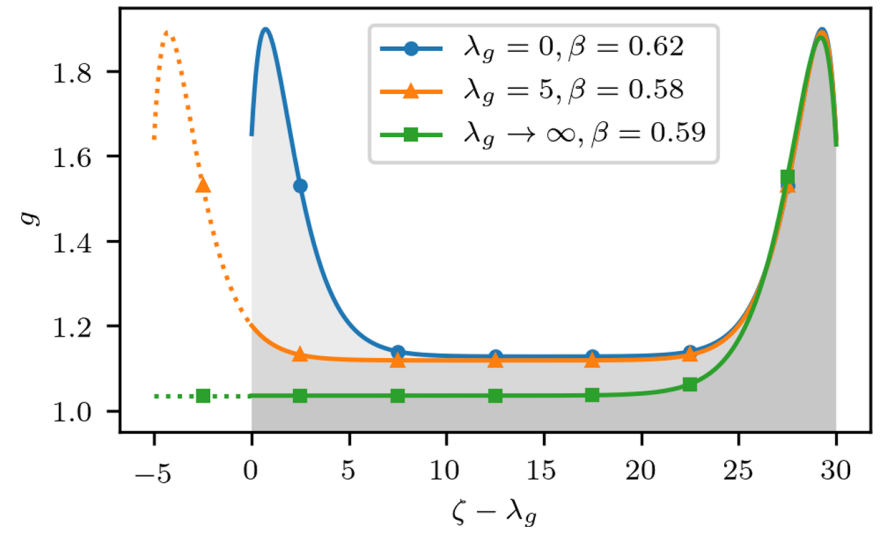

FIG. 7. Current profiles for probes with and without guard, for $\lambda_{p}=30$ and $\eta=10$.

We emphasize, however, that the dotted line for $\lambda_{g}=5$ is not representative for the actual current profile throughout the guard, since the leftmost end of the guard is not free but instead attached to some other carrier object, probably with another voltage, as indicated in Fig. 1. This alters the current profile, possibly also extending the edge effects, especially if the voltage difference is large between the probe and the carrier. If significant edge effects from the carrier object extends into the probe, the model presented herein may no longer apply. It is therefore important to have a sufficiently long guard, which leads us to the second method of accounting for a guard.

If we define an ideal guard as one which lets no edge effects extend into the probe whatsoever, the end effects may be eliminated from the profile function by removing the second term $\tilde{h}(\zeta)$ from Eq. (12), and use the fitting coefficients for a very long probe. This is, in fact, the same as letting $\lambda_{g} \rightarrow \infty$, and is also illustrated in Fig. 7. This approach will not include trace edge effects for a nonideal guard, which in any case, may not be representative.

As in Sec. V A, this has been evaluated for a sweep of voltages $\eta \in[10,100]$, and used to estimate the $\beta$ parameter, as indicated in the figure. The guard is seen to lower $\beta$ a bit compared to a free probe, but not by much. $\beta$ is similar for the two approaches of including a guard, to within the accuracy of the model. Our results support previous findings [14,16] that cylindrical Langmuir probes must be much more than ten Debye lengths in order to make end effects negligible, even with an ideal guard. Too short a guard may have nontrivial effects on the characteristics, and $\beta$ in particular, and is not covered by our model.

\section{Inferring plasma parameters}

Langmuir probes are used to infer plasma parameters such as the electron density by inverting the current-voltage characteristics for a set of measured currents at known bias voltages with respect to for instance a spacecraft or a rocket $[1,2,9,13]$. As an example, several recent space missions have used the fixed-bias m-NLP instrument [9-12], where a few cylindrical probes at different, positive voltages are used to measure $d I^{2} / d V$, and OML theory is used to make predictions on the electron density. According to OML theory, $d I^{2} / d V$ is proportional to the electron density, and independent of other unknowns, such as ion density, electron and ion temperatures, and the floating potential. However, if an inaccurate currentvoltage characteristic is assumed, for instance neglecting finite-length effects, it will necessarily lead to errors in the measurements. Using a more accurate, finite-length characteristic should alleviate these errors. While this is left as a future study, two ways of doing this are envisaged.

Consider a set of measured currents $\left\{I_{p}\right\}$ collected by probes $p$ with known voltages $\left\{V_{0 p}\right\}$ with respect to some common ground, i.e., the unknown floating potential $V_{0}$ of a spacecraft. In addition to the voltage, the probe current can be considered a function of plasma parameters such as the electron density and temperature, $I\left(V_{0}+V_{0 p} ; n, T\right)$. One may then find the parameters $\left(n, T, V_{0}\right)$ by minimizing the mean squared deviation between measured currents $I_{p}$ and model predictions $I\left(V_{0}+V_{0 p} ; n, T\right)$ for all $p$. This requires the problem to have a unique solution. In particular, the characteristic must be sufficiently sensitive to all parameters involved. Similar methods have been considered where an analytical expression is available for $I\left(V_{0}+V_{0 p} ; n, T\right)[13,16,35]$, but we remark that the method may be feasible even without one. It is sufficient for minimization algorithms to have a single callable function, and this function may very well interpolate between coefficients internally.

It may be difficult to arrive at a sufficiently robust and efficient inference algorithm by means of minimization. A promising alternative, avoiding the inversion problem altogether, is to use the measured currents $\left\{I_{p}\right\}$ as an input to a machine learning network or multivariate kriging regression, which is trained to predict plasma parameters such as the density. Such an approach has already been investigated for synthetic data [36]. For further studies along these lines, it would be natural to do comparisons with existing analysis techniques on real data.

It should also be remembered that a cylindrical probe attached to a low Earth orbit satellite moving with velocity $\mathbf{v}_{d}$ through a geomagnetic flux density $\mathbf{B}$ would be affected by an induced motional potential gradient $\mathbf{v}_{d} \times \mathbf{B}$, that would in particular deteriorate measurements by long probes [5]. This variation of potential would have a similar "energy smearing" effect as probe contamination. For example, assuming an orbital speed $v_{d} \approx 7500 \mathrm{~m} / \mathrm{s}$, a magnetic flux density $B \approx$ $35 \mu \mathrm{T}$, the motional potential gradient would be $\sim 0.26 \mathrm{~V} / \mathrm{m}$. For a cylindrical probe of length $5 \mathrm{~cm}$ oriented perpendicularly to $\mathbf{B}$, however, this would amount to a potential smearing on a given probe of approximately $13 \mathrm{mV}$, which is small compared to typical probe bias voltages. On the other hand, if multiple fixed bias and well separated probes are used on a given satellite, depending on the distance between probes and their relative orientations with respect to $\mathbf{B}$, the motional potential differerence between probes could be significant, and would have to be accounted for in processing probe data.

\section{CONCLUSION}

Collected current profiles along thin finite-length cylindrical probes have been studied with a particular attention to end effects. Making use of Buckingham's $\pi$ theorem, the current profile along a probe was shown to depend on only five 
independent dimensionless parameters. By limiting our attention to probes with a radius smaller than the attracted-species Debye length $\lambda_{D}$, and assuming a large plasma parameter, $n \lambda_{D}^{3} \gg 1$, the number of dimensionless parameters was reduced from five to three, consisting of the normalized position along the cylinder $\zeta=z / \lambda_{D}$, the normalized probe length $\lambda=$ $l / \lambda_{D}$, and the normalized probe potential $\eta=-q V / k T$ with respect to background plasma. Simulations have been made to cover a broad range of parameters relevant to probes used on many recent satellites deployed in ionospheric plasmas $[11,12]$. Based on kinetic simulation results for the normalized current collected per unit length $g(\zeta)$, as a function of the normalized axial position $\zeta$, it was possible to construct accurate empirical fits involving coefficients that can be interpolated in parameter space, in order to predict magnitudes and profiles of collected currents for arbitrary values $\zeta, \lambda$, and $\eta$ within the range of parameters considered. The empirical formula derived, along with the fitted parameters have also been found to predict total collected currents with excellent accuracy. Our main result is the quantification and parametrization of the current collected from the attracted species along thin finite-length cylindrical probes. The reported parametrization can be interpolated and used to calculate current collected by positive, fixed-bias multineedle Langmuir probes used on several satellites. For short probes, the overlap between this enhancement at the two ends, leads to a single maximum in $g(\zeta)$. As the normalized length of a probe increases however, the two end effects separate, and $g(\zeta)$ exhibits a distinctive two-hump profile, with maxima approximately one Debye length from either end. For long probes, sufficiently far from these maxima, the collected current per unit length accurately reproduces currents reported by Laframboise [2]. We show that for sufficiently large voltages $(\eta=-q V / k T>10)$, the current collected by a thin probe does scale approximately as $\eta^{\beta}$. For probes of practical lengths in ionospheric plasma conditions however, we find that $\beta$ is typically larger than the 0.5 value predicted with OML theory, for an infinite probe. This has implications, for example, in the proposed use of fixed-bias needle probes on satellites to infer plasma electron density independently of the temperature [9]. Indeed, referring to Eq. (18), it can be seen that with $0.5<\beta<$ 1 , the derivative of $I^{2}$ with respect to $V$ is proportional to $n^{2} V^{2 \beta-1} / T^{2 \beta-1}$, and with $\beta>0.5$, this derivative depends on density, the temperature, as well as on the probe voltage. The inference of the density based on the OML $\beta=0.5$ must therefore lead to discrepancies at levels depending on these three parameters. Extending our analysis to correct this predicament is beyond the scope of the present study, but we believe that the characterization of finite-length probe characteristics presented here provides the needed tools to better interpret fixed-bias multi-needle probe measurements in terms of plasma density and temperature.

We note in closing that our analysis was based on several simplifying assumptions, and that we recognize that it does not answer all questions concerning current collection with a cylindrical probe. It was assumed that finite-radius effects were negligible for radii less than the Debye length, which must according to Laframboise be the case in the middle of long probes [2]. A secondary finite-radius effect may, however, still exist within a few radii of either end. Considering space applications, the assumption of a Maxwellian background distribution for electrons is well justified by the fact that mid latitude ionospheric plasma is sufficiently collisional for the electron distribution to be near Maxwellian. The neglect of a drift electron velocity is also justified by the fact that the electron thermal speed is generally much larger than the ram speed of low Earth orbit (LEO) satellites and ionospheric winds. As for the zero magnetic flux density, with ionospheric electron thermal Larmor radii of order of a few centimeters, geomagnetic flux densities will likely affect current characteristics and collected current profiles. In addition, as pointed out by Brace [5], a cylindrical probe attached to a low Earth orbit satellite moving through a geomagnetic flux density would be affected by an induced motional potential gradient leading to "energy smearing." In future work our analysis analysis could be repeated with a magnetic flux density included. It would then become considerably more complex, as two nontrivial parameters consisting of the magnitude of the magnetic flux density, and angle with respect to the probe axis, would have to be taken into account. Buckingham's $\pi$ theorem would then lead to $i(z)$ depending on five parameters, instead of the three in Eq. (9). Kinetic simulations covering a broad range of relevant parameter space would then be significantly more computer intensive. This could be carried out for specific missions operating in restricted space environment conditions. This work should be considered in future studies. Additional complexities could be considered, such at those associated with the proximity of other satellite components and their detailed geometry, but detailed analyses of such cases should be made for specific missions, well ahead of deployment in space.

\section{ACKNOWLEDGMENTS}

This work was supported in part by the 4DSpace Strategic Research Initiative at the University of Oslo and the Natural Sciences and Engineering Research Council of Canada (NSERC). Simulation results presented here made use of the Compute Canada computing infrastructure as well as the Abel Cluster, owned by the University of Oslo and Uninett/Sigma2, and operated by the Department for Research Computing at USIT, the University of Oslo ITdepartment, Notur project number NN9299K. In particular, Sigvald Marholm would like to thank Wojciech J. Miloch and the Department of Physics at the University of Oslo for granting extended funding to support this work. He is also grateful for many fruitful discussions with Marianne Bakken on regression techniques, and for being recommended the excellent book by Hastie et al. [24].
[1] H. M. Mott-Smith and I. Langmuir, The theory of collectors in gaseous discharges, Phys. Rev. 28, 727 (1926).
[2] J. G. Laframboise, Theory of spherical and cylindrical langmuir probes in a collisionless, maxwellian plasma at rest, Ph.D. thesis, University of Toronto, 1966. 
[3] J.-P. Lebreton, S. Stverak, P. Travnicek, M. Maksimovic, D. Klinge, S. Merikallio, D. Lagoutte, B. Poirier, P.-L. Blelly, Z. Kozacek, and M. Salaquarda, The ISL Langmuir probe experiment processing onboard demeter: Scientific objectives, description and first results, Planet. Space Sci. 54, 472 (2006), first Results of the DEMETER Micro-Satellite.

[4] D. Knudsen, J. Burchill, S. Buchert, A. Eriksson, R. Gill, J.-E. Wahlund, L. Åhlén, M. Smith, and B. Moffat, Thermal ion imagers and Langmuir probes in the swarm electric field instruments, J. Geophys. Res.: Space Phys. 122, 2655 (2017).

[5] L. H. Brace, Langmuir probe measurements in the ionosphere, Geophysical Monograph-American Geophysical Union 102, 23 (1998).

[6] M. Rother, K. Schlegel, H. Lühr, and D. Cooke, Validation of champ electron temperature measurements by incoherent scatter radar data, Radio Sci. 45, 1 (2010).

[7] C. S. MacLatchy, C. Boucher, D. A. Poirier, and J. Gunn, Gundestrup: A Langmuir/mach probe array for measuring flows in the scrape-off layer of tdev, Rev. Sci. Instrum. 63, 3923 (1992).

[8] D. Johanning, W. Seifert, and A. Best, Analytical density correction for cylindrical Langmuir probes showing end effects, Plasma Phys. Controlled Fusion 27, 159 (1985).

[9] K. S. Jacobsen, A. Pedersen, J. I. Moen, and T. A. Bekkeng, A new Langmuir probe concept for rapid sampling of space plasma electron density, Meas. Sci. Technol. 21, 085902 (2010).

[10] T. A. Bekkeng, A. Barjatya, U.-P. Hoppe, A. Pedersen, J. I. Moen, M. Friedrich, and M. Rapp, Payload charging events in the mesosphere and their impact on Langmuir type electric probes, Ann. Geophys. 31, 187 (2013).

[11] H. Hoang, L. B. N. Clausen, K. Røed, T. A. Bekkeng, E. Trondsen, B. Lybekk, H. Strøm, D. M. Bang-Hauge, A. Pedersen, A. Spicher, and J. I. Moen, The multi-needle Langmuir probe system on board norsat-1, Space Sci. Rev. 214, 75 (2018).

[12] T. A. Bekkeng, E. S. Helgeby, A. Pedersen, E. Trondsen, T. Lindem, and J. I. Moen, Multi-needle Langmuir probe system for electron density measurements and active spacecraft potential control on cubesats, IEEE Trans. Aerosp. Electron. Syst. 55, 2951 (2019).

[13] H. Hoang, K. Røed, T. A. Bekkeng, J. I. Moen, A. Spicher, L. B. N. Clausen, W. J. Miloch, E. Trondsen, and A. Pedersen, A study of data analysis techniques for the multi-needle Langmuir probe, Meas. Sci. Technol. 29, 065906 (2018).

[14] A. R. Hoskinson and N. Hershkowitz, Effect of finite length on the current voltage characteristic of a cylindrical Langmuir probe in a multidipole plasma chamber, Plasma Sources Sci. Technol. 15, 85 (2006).

[15] E. F. Jaeger, L. A. Berry, and D. B. Batchelor, Characteristics of a cylindrical Langmuir probe of finite length, J. Appl. Phys. 69, 6918 (1991).

[16] S. Marholm, R. Marchand, D. Darian, W. J. Miloch, and M. Mortensen, Impact of miniaturized fixed-bias multineedle Langmuir probes on cubesats, IEEE Trans. Plasma Sci. 47, 3658 (2019).
[17] H. Amemiya, K. Oyama, and K. Hirao, Observation of the energy distribution of thermal electrons in the midlatitude ionosphere, Planet. Space Sci. 33, 875 (1985).

[18] T. Abe, K.-i. Oyama, H. Amemiya, S. Watanabe, T. Okuzawa, and K. Schlegel, Measurements of temperature and velocity distribution of thermal electrons by the akebono (exos-d) satellite, J. Geomagn. Geoelectr. 42, 537 (1990).

[19] E. Buckingham, On physically similar systems; illustrations of the use of dimensional equations, Phys. Rev. 4, 345 (1914).

[20] H. L. Pécseli, Waves and Oscillations in Plasmas (CRC Press, Boca Raton, FL, 2012).

[21] R. Marchand, PTetra, a tool to simulate low orbit satelliteplasma interaction, IEEE Trans. Plasma Sci. 40, 217 (2011).

[22] R. Marchand and P. A. R. Lira, Kinetic simulation of spacecraft-environment interaction, IEEE Trans. Plasma Sci. 45, 535 (2017).

[23] C. Geuzaine and J.-F. Remacle, Gmsh: A 3-d finite element mesh generator with built-in pre- and post-processing facilities, Int. J. Numer. Methods Eng. 79, 1309 (2009).

[24] T. Hastie, R. Tibshirani, and J. Friedman, The Elements of Statistical Learning: Data Mining, Inference, and Prediction, Second Edition, Springer Series in Statistics (Springer, New York, 2009).

[25] W. S. Cleveland, Robust locally weighted regression and smoothing scatterplots, J. Am. Stat. Assoc. 74, 829 (1979).

[26] H. Ikezi, M. Fujiwara, and K. Takayama, Probe noise in quiescent plasmas, J. Phys. Soc. Jpn. 25, 1663 (1968).

[27] P. Horowitz and W. Hill, The Art of Electronics (Cambridge University Press, NY, USA, 2015).

[28] S. Marholm and R. Marchand, 99 probes, doi: 10.5281/zenodo.3269686 (2019).

[29] S. Marholm, sigvaldm/localreg 0.2.0, doi: 10.5281/zenodo.3234461 (2019).

[30] R. Marchand, Test-particle simulation of space plasmas, Commun. Comput. Phys. 8, 471 (2010).

[31] S. Marholm and D. Darian, langmuirproject/langmuir 0.4.0, doi:10.5281/zenodo.3268526 (2019).

[32] N. Schlömer, G. McBain, T. Li, V. M. Ferrándiz, eolianoe, L. Dalcin, nilswagner, L. Schwarz, J. Blechta, C. Coutinho et al., nschloe/meshio: 2.3.3, doi:10.5281/zenodo.1173115 (2020).

[33] E. Jones, T. Oliphant, P. Peterson et al., SciPy: Open source scientific tools for Python, 2001 (2016); P. Virtanen, R. Gommers, T. E. Oliphant, M. Haberland, T. Reddy, D. Cournapeau, E. Burovski, P. Peterson, W. Weckesser, J. Bright et al., SciPy 1.0: fundamental algorithms for scientific computing in Python, Nat. Meth. 17, 261 (2020).

[34] E. Süli and D. Mayers, An Introduction to Numerical Analysis (Cambridge University Press, Cambridge, New York, 2003).

[35] D. Darian, S. Marholm, M. Mortensen, and W. J. Miloch, Theory and simulations of spherical and cylindrical Langmuir probes in non-maxwellian plasmas, Plasma Phys. Controlled Fusion 61, 085025 (2019).

[36] J. Chalaturnyk and R. Marchand, A first assessment of a regression-based interpretation of Langmuir probe measurements, Frontiers Phys. 7, 63 (2019). 